\title{
Adaptive and Blind Wideband Spectrum Sensing Scheme Using Singular Value Decomposition
}

\author{
Zhuhua Hu, ${ }^{1,2}$ Yong Bai, ${ }^{1,2}$ Yaochi Zhao, ${ }^{1}$ Chong Shen,,2 and Mingshan Xie ${ }^{1}$ \\ ${ }^{1}$ College of Information Science \& Technology, Hainan University, Haikou, China \\ ${ }^{2}$ State Key Laboratory of Marine Resource Utilization in the South China Sea, Hainan University, Haikou, China \\ Correspondence should be addressed to Yong Bai; bai@hainu.edu.cn
}

Received 15 August 2017; Revised 12 November 2017; Accepted 7 December 2017; Published 25 December 2017

Academic Editor: Waleed Ejaz

Copyright (c) 2017 Zhuhua Hu et al. This is an open access article distributed under the Creative Commons Attribution License, which permits unrestricted use, distribution, and reproduction in any medium, provided the original work is properly cited.

\begin{abstract}
The Modulated Wideband Converter (MWC) can provide a sub-Nyquist sampling for continuous analog signal and reconstruct the spectral support. However, the existing reconstruction algorithms need a priori information of sparsity order, are not self-adaptive for SNR, and are not fault tolerant enough. These problems affect the reconstruction performance in practical sensing scenarios. In this paper, an Adaptive and Blind Reduced MMV (Multiple Measurement Vectors) Boost (ABRMB) scheme based on singular value decomposition (SVD) for wideband spectrum sensing is proposed. Firstly, the characteristics of singular values of signals are used to estimate the noise intensity and sparsity order, and an adaptive decision threshold can be determined. Secondly, optimal neighborhood selection strategy is employed to improve the fault tolerance in the solver of ABRMB. The experimental results demonstrate that, compared with ReMBo (Reduce MMV and Boost) and RPMB (Randomly Projecting MMV and Boost), ABRMB can significantly improve the success rate of reconstruction without the need to know noise intensity and sparsity order and can achieve high probability of reconstruction with fewer sampling channels, lower minimum sampling rate, and lower approximation error of the potential of spectral support.
\end{abstract}

\section{Introduction}

Spectrum resource has become increasingly scarce with emerging wireless services. Nevertheless, assigned radio spectrum to authorized users is mostly underutilized. Cognitive radio (CR) technology can improve frequency spectrum utilization by detecting and accessing the frequency range that has not been occupied by authorized users (primary users). Therefore, one of the crucial tasks in the cognitive radio system is to constantly monitor the potential spectrum bands and detect the activities of primary users $[1,2]$. The methods of signal detection on narrow band mainly include energy detection [3], coherent detection [4], and feature detection [5]. Since the wideband spectrum sensing can provide more spectrum access opportunities for cognitive users (secondary users), multiband based wideband spectrum sensing has gained much more research attention in recent years $[6,7]$.

At the receiving side, the traditional method of acquiring RF (Radio Frequency) information is to use demodulation under the condition of known carrier. However, in practical scenarios, it is often required to directly perform blind sensing for high frequency wideband analog signals, which brings great challenges to spectrum detection. Firstly, extremely high sampling rate has exceeded the limit of physical ADC (Analog-to-Digital Converter) capability. Meanwhile, the storage and transmission for the sampled data can bring huge overhead. In addition, the carrier frequency of the received multiband RF signal is usually unknown. To address these challenges, compressed sensing theory is applied to wideband spectrum sensing based on compressed sampling and signal reconstruction $[8,9]$. In order to perform sub-Nyquist sampling on continuous spectrum signal, Mishali et al. proposed the MWC (Modulated Wideband Converter) scheme which uses multichannel compressed sampling and reconstruction with parallel structure based on compressive sensing and time-frequency transform theory $[10,11]$. In MWC system, the multiband analog sparse signal can be collected with sub-Nyquist sampling, and the spectral support of signal can be reconstructed by a CTF (continuous-to-finite) block. 
From [10], it is known that the time domain reconstruction model of MWC can be attributed to the Multiple Measurement Vectors (MMV) problem. MWC has been considered to be an effective approach for compressed sampling and signal reconstruction with its applications on radar, broadband communication, and cognitive radio spectrum sensing [1214].

The accurate reconstruction of spectral support of signal is the key to realize the spectrum sensing. At present, there is still much room for improvement in the success rate of sensing, the required minimum number of channels, and the maximum number of subbands that can be reconstructed. The solvability of MMV problems is important for MWC reconstruction ability. To enhance the solvability of MMV problem, Reduce MMV and Boost (ReMBo) algorithm, which can transform MMV problem into single measurement vectors (SMV) problem is proposed in [15]. In ReMBo, the sampling value matrix $Y$ is projected on a random vector $w$ which is independently and identically distributed in the interval $[-1,1]$ and obtains a column vector $v, v=$ $Y w$. Then, it tries to obtain the estimated spectral support of signal by iteratively computing SMV problem $(v=$ $\Phi Z w=\Phi z)$ for many times. Although ReMBo is better than most of the existing reconstruction algorithms in the success rate of reconstruction and computational overhead, there is still a big gap between the maximum number of reconstructed frequency subbands and the theoretical upper limit [16]. The total sampling rate and the minimum number of required channels of ReMBo algorithm are still much higher than the theoretical lower bound [10,17]. To improve the performance of MWC system, a dimension adjustable Randomly Projecting MMV and Boost (RPMB) framework algorithm, which transforms the initial MMV problem into a series of low dimensional MMV problems with the same sparsity order, was proposed in [18]. In the solving process, the solution is tested several times until a satisfactory solution is obtained. Compared with ReMBo, RPMB can reduce the number of required hardware channels and improve the maximum number of reconstructed frequency subbands in the condition of precise reconstruction. In spite of this, the RPMB framework is still faced with difficult problems to be solved: (1) The reconstruction performance of RPMB is greatly reduced under low SNR because the influence of the noise intensity is not considered in the RPMB solver; (2) the RPMB is not flexible enough since the decision threshold of RPMB is a fixed value, which has no self-adaptive ability for the uncertainty of the noise; (3) RPMB needs to know the sparsity order of the signal in advance, which is difficult in some practical applications; (4) although the RPMB solver improves the algorithm's fault tolerance by finding more potential support bands, the effect of fault tolerance remains to be improved.

To address the above-mentioned problems, this paper proposes a Self-Adaptive and Blind Reduced MMV and Boost (ABRMB) scheme for MWC based on SVD [19]. Our main contributions are summarized as follows.

(1) ABRMB can first reveal the intrinsic properties of signal using SVD and perform the linear fitting on the noise singular value by using the tail singular values as noise data.
Then, it can estimate the noise intensity, and the threshold of deciding support elements can be set adaptively.

(2) Our proposed scheme calculates the contribution of the estimated noise singular on the singular values of signal and figures out the sparsity order by using gradient and difference operation. Hence the number of signal subbands can be estimated.

(3) In the solver of ABRMB, the fault tolerance can be improved by the optimal neighborhood selection method. Compared with ReMBo and RPMB, when noise intensity and sparsity order are unknown, our proposed scheme can use fewer channels to achieve the high probability reconstruction of spectral support in low SNR range and increase the maximum subband number of reconstructed signal.

The rest of this paper is organized as follows. In Section 2, the related work is introduced. In Section 3, the multiband sparse signal model is introduced, and sampling principles of MWC are described as well as some of the present major issues. Section 4 introduces the key ideas of the noise intensity estimation, sparsity order estimation, and optimal neighborhood selection. In Section 5, the ABRMB scheme includes its solver which are specifically described, the convergence proof of which is also given. Section 6 performs validation and analysis on ABRMB from four important performance evaluation indexes. Conclusions and discussion are in Section 7.

The main notations used in this paper are listed in Notations to make them clearer and easier to read.

\section{Related Work}

Current research on the performance of spectrum sensing can be mainly classified into the following two categories. The first category, especially in the design of CRN, focuses on investigating the optimal trade-off between energy consumption and throughput for secondary users (SUs). The second category, especially for wideband spectrum sensing, mainly focuses on studying how to achieve a good balance between noise interference and sensing accuracy.

As for the first category, some earlier researches focus on the trade-off between the interference and throughput. Specifically, for the unslotted CRN, Yang et al. [20] designed an interference-constrained novel simultaneous sensing and transmission scheme. This scheme exploited the statistic information of the activities of primary users (PUs), and the transmission duration is adaptively adjusted to avoid the interference. In the same period, energy efficient techniques, in cooperative spectrum sensing (CSS), have also gained a lot of attention [6]. In [21], considering the energy consumption problems of each sensing node in cooperative CR sensor network, a user selection scheme was proposed to minimize the energy consumed on each CR node based on the binary knapsack problem and its dynamic solution. After that, Ejaz et al. [22] indicated that an optimal sensing, reporting, and transmission duration can be found while providing a given throughput and reliability constraint. The solution can obtain the best trade-off between energy consumption and throughput for SUs. 
(a) Sparse multiband signal model

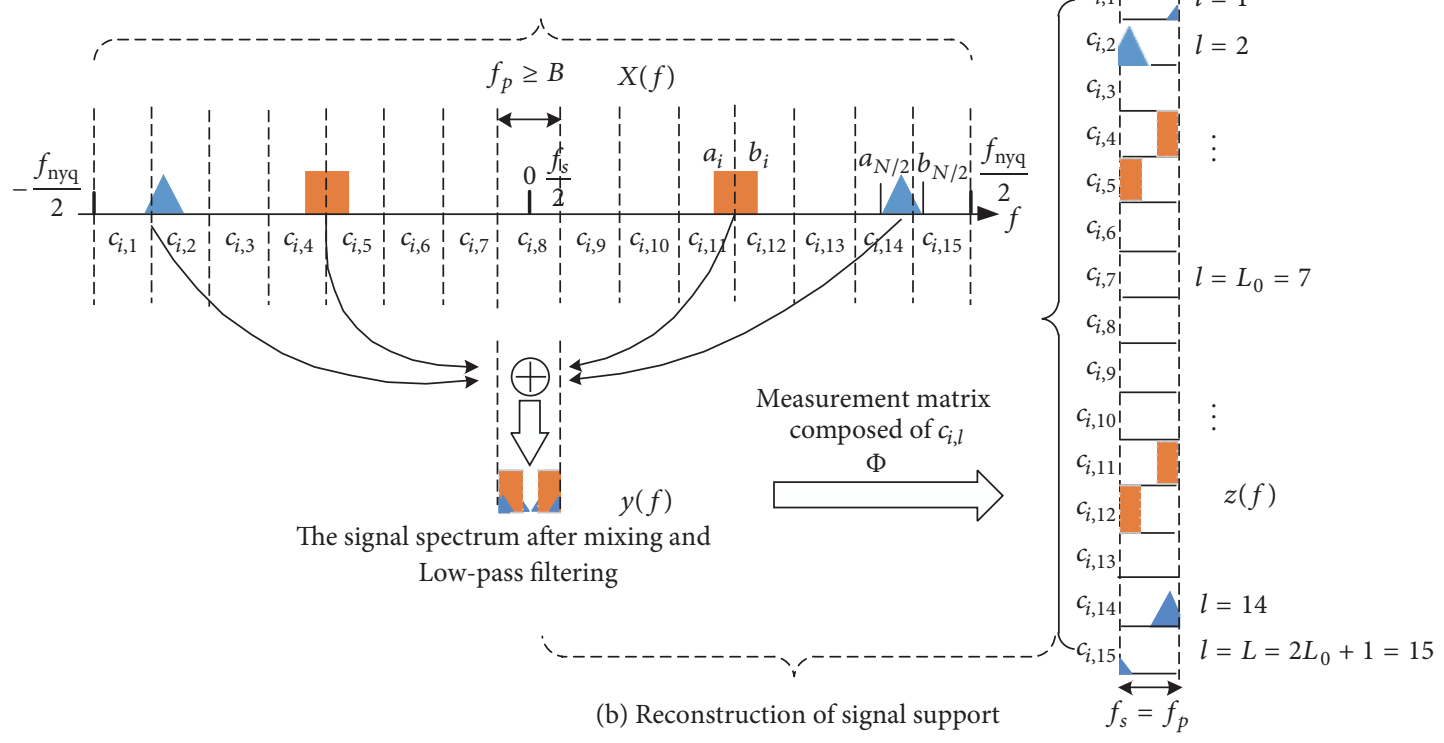

FIGURE 1: The reconstruction principles of the spectral support of MWC system.

As for the second category, in order to overcome the limitation of hardware technology and achieve wideband spectrum sensing, the research work mainly concentrated on how to get a good trade-off between the noise interference and sensing accuracy [4]. Firstly, based on the compressed sensing theory, a parallel MWC architecture for wideband spectrum sensing was designed by Mishali et al. [10,11], which can achieve sub-Nyquist sampling for the wideband signal. Then, a MWC system with SwSOMP algorithm was proposed in [23]. Given the noise interference, the algorithm can get higher reconstruction precision of spectral support. In [24], in order to reduce the high hardware complexity of MWC, a compressive wideband spectrum sensing scheme based on a single channel was designed.

The work of this paper belongs to the second category. All of the aforementioned literatures only consider ideal noise interference model (NIM) and assume that the signal sparsity is known. However, in reality, NIM is always nonideal, where the intensity of noise varies with the change of communication environment. Moreover, the signal sparsity is always unknown in reality. In addition, we also hope to adopt a better reconstruction strategy and use fewer parallel channels to obtain higher reconstruction accuracy. Therefore, these problems need to be further investigated.

\section{Problem Statement of MWC Model and Our Proposed Problem Solution}

3.1. MWC System Model. The reconstruction principles of the spectral support of MWC system is shown in Figure 1. Figure 1(a) shows the sparse multiband analog signal model. Sparse multiband analog signal is common in cognitive radio [25]. Suppose the received signal $x(t)$ is a sparse bandpass analog signal. The spectrum is distributed in frequency interval $\left[-f_{\text {nyq }} / 2, f_{\text {nyq }} / 2\right]$, and $f_{\text {nyq }}$ is Nyquist rate, which usually can reach GSPS order of magnitude. Suppose the spectrum of $x(t)$ only contains $N$ subbands whose bandwidth is $B_{i} \leq B(N \geq i>0)$, and there is no overlap between subbands. $B$ is the maximal bandwidth in subbands, and the center carrier frequency of each subband is unknown. $P_{N}$ and $B$ can be defined as

$$
\begin{aligned}
P_{N} & =\bigcup_{i=1}^{N / 2}\left\{\left(a_{i}, b_{i}\right) \cup\left(-b_{i},-a_{i}\right)\right\}, \\
B & =\max _{i}\left(b_{i}-a_{i}\right) .
\end{aligned}
$$

In (1), $P_{N}$ is union of all subbands whose amplitude is not zero, which represents the effective frequency component of $x(t)$.

As shown in Figure 1(a), the whole band is equally divided into $L$ consecutive narrow band channels, every bandwidth of which is no less than $B$. Then the spectrum of $x(t)$ has at most $2 N$ parts which get energy in the whole frequency band. If the channel is marked $[1, \ldots, L]$, the set of channel numbers corresponding to each subband $X_{i}(f)$ is the spectral support of $x(t)$, which is defined as $\Lambda=\operatorname{supp}(X(f)) .|\Lambda|$ is the number of nonzero elements in $\Lambda$, that is, the potential of $\Lambda$. The frequency bands corresponding to these numbers are called support bands. Since $2 N$ is much smaller than $L, x(t)$ can be considered as sparse multiband signal. Figure 1(b) shows the reconstruction process of the support of $x(t)$. Assume that the number of bands is $4, f_{s}=f_{p}$, and $f_{p} \geq B$. We divide the wideband spectrum into $L$ spectrum slices, where $L=2 L_{0}+1$. In order to ensure that the discrete Fourier transformation result of sampling sequence contains all the components of the original signal spectrum $X(f), L_{0}$ must satisfy $L_{0}=\left\lfloor\left(f_{\text {nyq }}+f_{s}\right) / 2 f_{p}\right\rfloor-1$. After mixing and lowpass filtering, the spectrum information of the original signal appears in the sampling interval $\left[-f_{s} / 2, f_{s} / 2\right]$, and the mixing 
coefficient of each spectrum slice is $c_{i l}$, where $l$ is the index of spectrum slices. According to the theory of compressed sensing, we can obtain the spectrum support of the multiband signal.

In conclusion, the support bands of $x(t)$ must meet two conditions: (1) it is distributed in an extremely wide frequency range; (2) the support bands of signal only exist in a few discrete frequency spectrum.

3.2. Sampling in MWC System. MWC contains multiple parallel sampling channels, and each channel has same structure with mixer, low-pass filter, and ADC [10]. The received signal $x(t)$ is input to $m$ parallel channels at the same time, and each channel is multiplied by periodic mixing signal $p_{i}(t)$ with different mode, which makes spectrum of $x(t)$ move to baseband. Each of the channels' $p_{i}(t)$ is not related. The period of $p_{i}(t)$ is $T_{p}=1 / f_{p}$, and $M$ is used to show the number of random \pm 1 switches in a cycle. $M f_{p}$ is defined as the switching frequency of mixed signals. The mixed signals pass through the low-pass filter whose cut-off frequency is $1 / 2 T_{s}$. At last, it passes through ADC whose sampling rate is $f_{s}=1 / T_{s}$ and obtains $m$ groups low-speed digital sampling sequences $y_{i}[n]$.

On analysis of the $i$ th channel, the Fourier series expansion of the random mixing function is

$$
p_{i}(t)=\sum_{l=-\infty}^{\infty} c_{i l} e^{j 2 \pi f_{p} l t},
$$

where $c_{i l}=d_{l} \sum_{k=0}^{L-1} \alpha_{i k} e^{-j(2 \pi / L) l k}, \alpha_{i k} \in\{-1,+1\}$. When $l=0$, $d_{0}=1 / L$, and when $l \neq 0, d_{l}=\left(1-e^{-j(2 \pi l / L)}\right) / j 2 \pi l$. In $(2)$, $p_{i}(t)$ denotes a pseudorandom sequence of \pm 1 , which is used as a mixing signal of the $i$ th sampling channel; $l$ is index of spectrum slice; $c_{i l}$ is the coefficient of Fourier series expansion.

Next, after passing through the low-pass filter with frequency characteristic of $H(f)=\left\{\begin{array}{l}1|f| \leq f_{s} / 2 \\ 0|f|>f_{s} / 2\end{array}\right.$, the relationship between DTFT (Discrete Time Fourier Transform) of $y_{i}[n]$ and the Fourier transform $X(f)$ of $x(t)$ is

$$
Y_{i}\left(e^{j 2 \pi f T_{s}}\right)=\sum_{l=-L_{0}}^{L_{0}} c_{i l} X\left(f-l f_{p}\right) .
$$

In (3), $f \in\left[-f_{s} / 2, f_{s} / 2\right]$, and $L_{0}$ is the smallest integer that can satisfy $L=2 L_{0}+1 \geq f_{\text {nyq }} / f_{p}$. Formula (3) shows that the spectrum of the output sequence $y_{i}[n]$ is equivalent to the shift weighted sum of the original signal spectrum $X(f)$ with $f_{p}$ as its step, which is intercepted into spectral fragments with $f_{s}$ width by low-pass filter. If $Y_{i}\left(e^{j 2 \pi f T_{s}}\right)$ is considered as the $i$ th component of m-dimensional column vector $y(f)$ and $X\left(f-l f_{p}\right)$ is considered as the $l$ th component of $2 L_{0}+1$ dimensional column vector $z(f)$, then (3) can be expressed as

$$
y(f)=\Phi z(f), \quad f \in\left[-\frac{f_{s}}{2}, \frac{f_{s}}{2}\right]
$$

In (4), $\Phi$ is a $m \times L$ measurement matrix, $\Phi_{i l}=c_{i,-l}=$ $c_{i l}{ }^{*}, 1 \leq i \leq m$, and $m<L$. If IDTFT (Inverse Discrete Time
Fourier Transform) is performed on both ends of (4), we can get the corresponding relationship between the sequence $Z[n]=\left[z_{1}[n], z_{2}[n], \ldots, z_{L}[n]\right]^{T}$ and the sampling data $Y[n]=\left[y_{1}[n], y_{2}[n], \ldots, y_{m}[n]\right]^{T}$.

$$
Y[n]=\Phi Z[n]
$$

For any frequency $f \in\left[-f_{s} / 2, f_{s} / 2\right],(5)$ is a typical compressed sensing problem. When sampled values matrix $Y$ and an observation matrix $\Phi$ are known, a sparse vector $Z$ can be recovered. Since MWC is a MMV problem, the one-dimensional measured value vector turns into a twodimensional matrix. As a result, paper [15] gets a onedimensional vector after projecting $Y$ ingeniously and then reconstructs the signal support bands by using compressed sensing technique. Furthermore, the reconstruction by building a CTF (continuous-to-finite) module is described in [10].

3.3. Problem Statement. The principle of reconstructing spectral support for MWC is shown in Figure 1 where Figure 1(b) gives a specific reconstruction process. As can be seen in Figure 1, if the number of support bands of $x(t)$ is $N$, the maximum reconstructed subbands are $2 \mathrm{~N}$ after passing through the MWC system; that is, the maximum possible sparsity order $K$ is $2 N$. In the MOMPMMV (Modified Orthogonal Matching Pursuit for MMV), the solver mentioned in paper [18], the initial solution $\ddot{Z}$ is obtained via the OMPMMV, which needs to know $K$ beforehand. In addition, in the condition judgment of RPMB, the algorithm uses $|\widehat{\Lambda}| \leq K$ to determine whether the potential of spectral support meets the requirements. However, in cognitive radio applications, it is difficult to know the sparsity order of signal. Therefore, aiming at the practical application, an effective method to estimate the sparsity order of signal is needed.

Secondly, in [18], the condition of deciding spectral support is $\left\|\widetilde{Z}^{i \rightarrow}\right\|_{2} \geq \varepsilon$; and the condition of determining whether the spectral support meets the requirements in the iterative process of RPMB is $\|\widehat{Y}-\Phi \widehat{Z}\|_{F} \leq \varepsilon$. As mentioned in Section 1, in the case where there is no noise or high SNR, $\varepsilon$ can be figured by taking a fixed small value. If the SNR is low or fluctuates greatly, the value of $\varepsilon$ will have to perform self-adaptive change according to the intensity of the noise. In paper [18], $\varepsilon$ is a fixed value without considering SNR. Hence, RPMB algorithm is neither suitable nor flexible under low or fluctuated SNR. Thus an effective method is needed to estimate the intensity of the noise and adaptively change the decision threshold $\varepsilon$.

Finally, in MOMPMMV, to improve the fault tolerance ability, the method is $\ddot{\Lambda}=\max \left(\left\|\ddot{Z}^{i \rightarrow}\right\|_{2}, m\right)$, where $m$ is the number of sampling channels, that is, taking $m$ maximum norm 2 as the initial spectral support. This method is effective, but it is not the best way. For example, as shown in Figure 1(a), the $N / 2$ th subband $X_{N / 2}(f)$ of $x(t)$ is located at the 14 th and 15 th narrow channels, and the spectrum energy of the 15th narrowband channel is quite small, as a result of which if the fault tolerance method in [18] is used, under low SNR, number 15 will not be able to merge into the initial support $\ddot{\Lambda}$. So it is necessary to design a better fault tolerance strategy. 


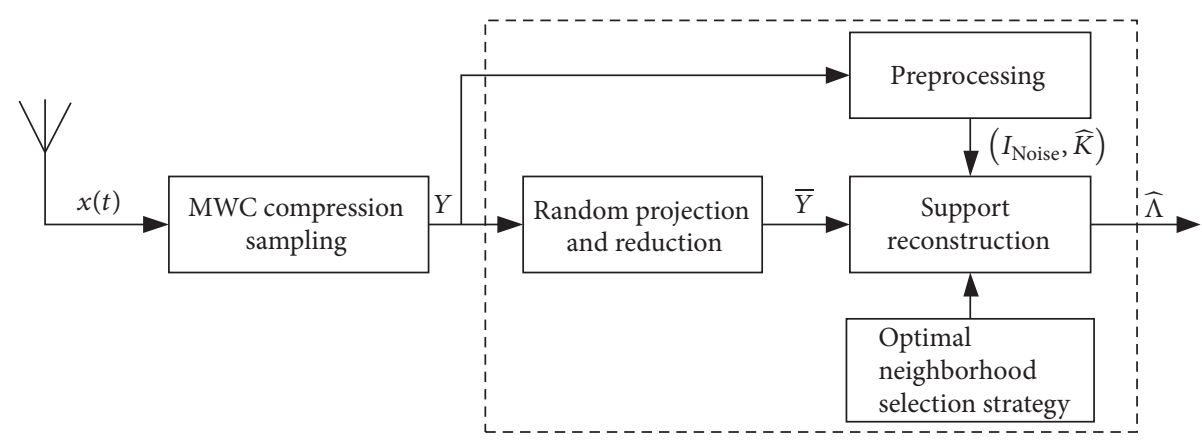

FIgURE 2: The flowchart of our proposed problem solution.

3.4. Brief Description of Our Proposed Problem Solution. Our proposed problem solution is shown in Figure 2 where multiband analog signal $x(t)$ passes through the MWC system to obtain compressed sampling data $Y$. Then, in order to reduce complexity, we reduce the dimension of $Y$ by means of random projection. Subsequently, the signal sparsity and noise intensity are estimated in the preprocessing, namely, $\widehat{K}$ and $I_{\text {Noise }}$. Meanwhile, to improve the fault tolerance ability, we adopt the optimal neighborhood selection strategy, which is described in detail in Section 4.3. Finally, by using the prior knowledge and the compression sensing reconstruction algorithm, we can obtain the estimated spectral support $\widehat{\Lambda}$. It is worth noting that, in the reconstruction algorithm, we can adaptively adjust the decision threshold by using the estimated noise intensity $I_{\text {Noise }}$.

\section{Preprocessing of ABRMB Scheme}

4.1. Estimation of Noise Intensity. Singular value decomposition is one of the most basic and important tools in modern numerical analysis. The SVD is performed on the measured values $Y$ with noise using (6). $Y \in \mathbb{R}^{m \times r}, m \geq i>0, r \geq j>$ 0 , and $m<r$, where $r$ is sampling length.

$$
Y=U \Sigma V^{T}
$$

In (6), unitary matrix $U \in \mathbb{R}^{m \times m}$ is the left singular vector of $Y$, and unitary matrix $V \in \mathbb{R}^{r \times r}$ is right singular vector. $\Sigma \in \mathbb{R}^{m \times r}$ is a diagonal matrix, and the main diagonal element is the singular value with a descending order. Since $\operatorname{rank}(Y)=$ $m$, (6) can be simplified as

$$
Y=U_{\downarrow m}\left(\begin{array}{cc}
\Sigma_{K} & 0 \\
0 & \Sigma_{m-K}
\end{array}\right) V_{\downarrow m}{ }^{T} .
$$

Let $\Sigma_{K}=\operatorname{diag}\left(\sigma_{1}, \sigma_{2}, \ldots, \sigma_{K}\right)$ and $\Sigma_{m-K}=\operatorname{diag}\left(\sigma_{K+1}\right.$, $\left.\sigma_{K+3}, \ldots, \sigma_{m}\right) . \sigma_{i}$ is $i$ th singular value of $Y$, and $m \geq i \geq 1$. After SVD, an important characteristic is that most of the energy of signal is concentrated in the first $K$ singular values, while the energy of the noise is distributed in all of the singular values; but noise can be reflected in the tail singular value. Assume that the singular value of $Y$ can be decomposed into the singular value $\Sigma_{s}$ of the original signal and the singular value $\Sigma_{n}$ of the noise, and $\Sigma_{s}=\operatorname{diag}\left(\sigma_{s 1}, \sigma_{s 2}, \ldots, \sigma_{s m}\right)$,
$\Sigma_{n}=\operatorname{diag}\left(\sigma_{n 1}, \sigma_{n 2}, \ldots, \sigma_{n m}\right)$. Figure 3 describes the contribution of $\Sigma_{s}$ and $\Sigma_{n}$ to $\Sigma$ under different SNR, which are represented by $R_{s}$ and $R_{n}$ respectively. The definitions are as follows:

$$
\begin{aligned}
& R_{s}=\frac{\Sigma_{s} \cdot}{\Sigma}=\bigcup_{i=1}^{m}\left(\frac{\sigma_{s i}}{\sigma_{i}}\right), \\
& R_{n}=\frac{\Sigma_{n} \cdot}{\Sigma}=\bigcup_{i=1}^{m}\left(\frac{\sigma_{n i}}{\sigma_{i}}\right) .
\end{aligned}
$$

The contribution of the original signal to the tail of the singular values is small. The energy of the signal is concentrated in the first $K$ singular values, and the tail of the singular values is mainly determined by noise. Figure 3 shows that the best data source for noise intensity estimation is bottom $50 \%$ of the singular values. Because the available singular values are also reduced with the decrease of the sampling channels, in order to ensure the accuracy of estimation, the bottom $30 \%$ of the singular values is adopted to estimate the noise intensity in this paper.

Figure 4 illustrates the variation of the singular values of $Y$ with different noise intensity and different sampling channel number. As shown in Figure 4, the singular value is larger when the SNR is lower or the sampling channels number is more. It should be noted that since the initial part of the singular value is mainly determined by the energy of the signal, the initial part of the singular value has little change versus different SNR and channel numbers. Yet the tail of the singular values changes a lot under different SNR because it is influenced significantly by noise intensity as the contribution of real signal is much less for those values compared with noise. This fact is utilized in this paper to estimate the noise intensity.

Next, the distribution of the noise singular values is investigated. Since the noise is the uniformly distributed white Gaussian noise (WGN), it can be seen in Figure 5, the singular values of noise are largely distributed on a straight line under the circumstances with different SNR and different sampling channel number. Using this fact, the singular values of tail $30 \%$ of $Y$ are used as singular values of noise to perform linear fitting. The fitting line is used to estimate the noise singular values, and the noise intensity is estimated by (9). The fitting result is shown in Figure 6. Since there is little 

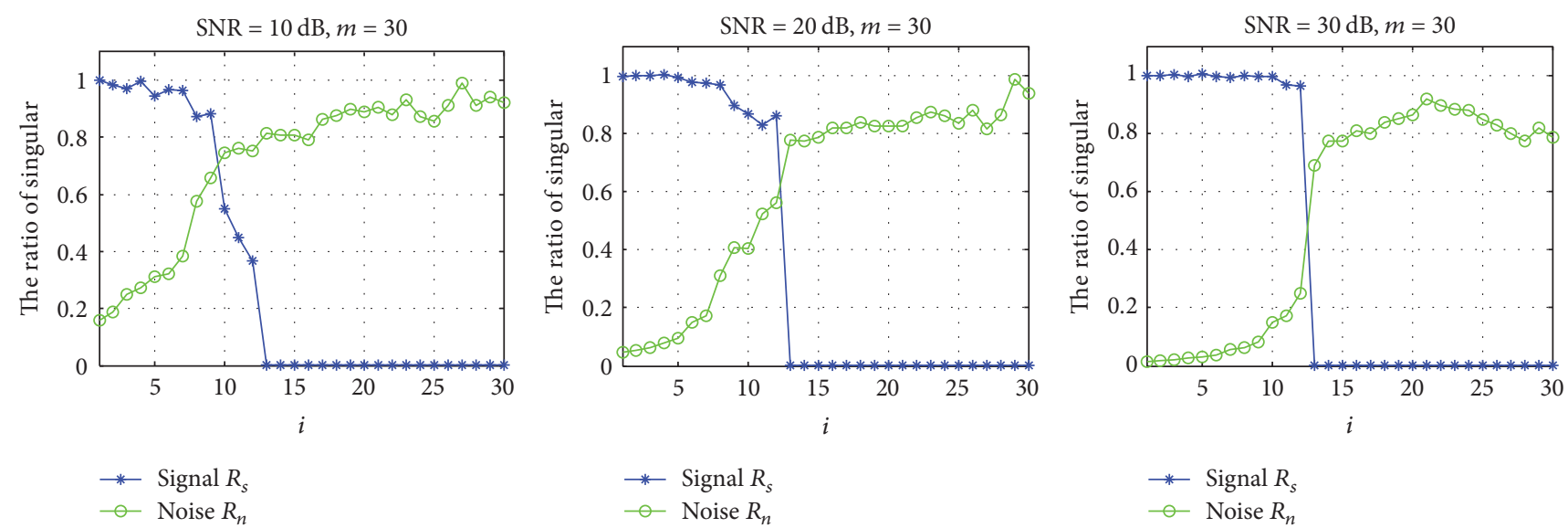

FIgURE 3: The contribution of the singular values of the signal and noise to total singular values.
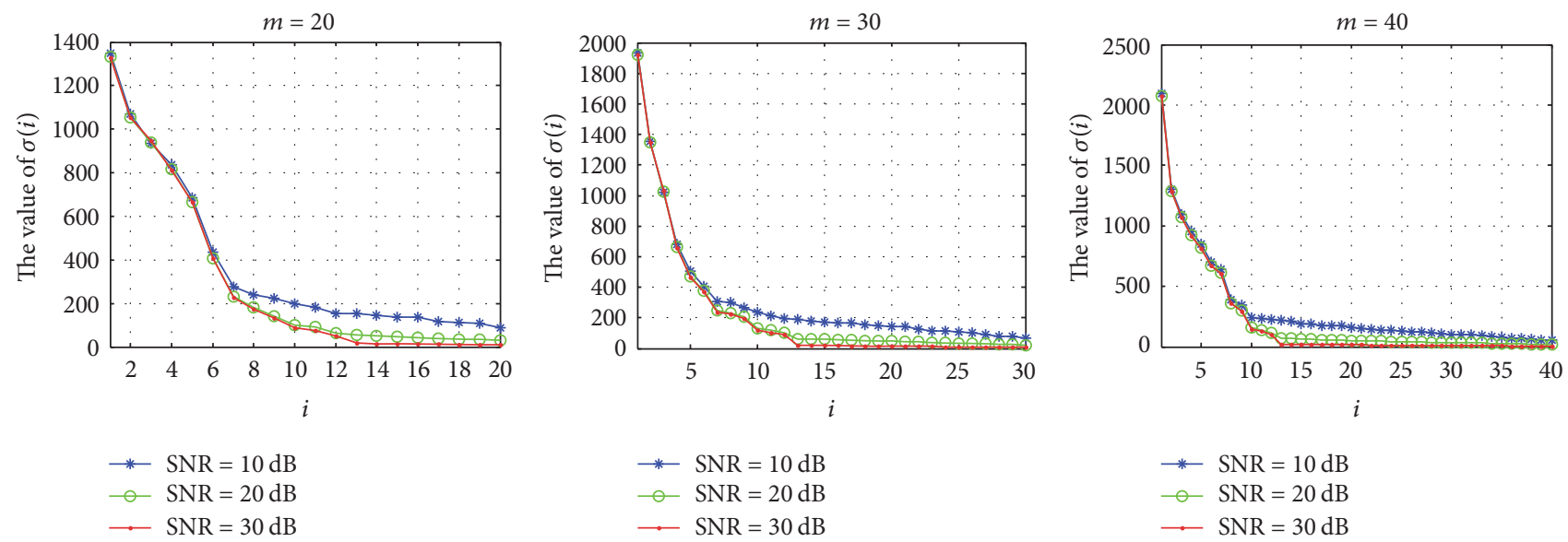

FIGURE 4: The comparison of singular value of $Y$ under different SNR.
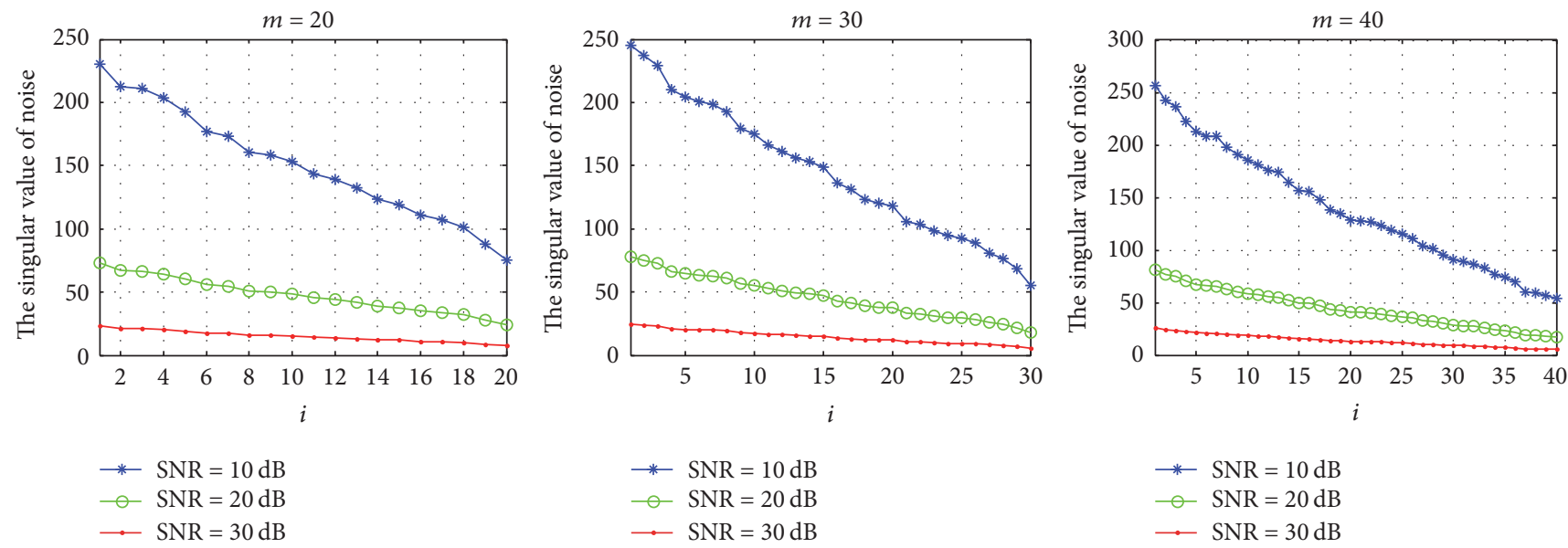

FIGURE 5: The distribution of noise singular values.

energy of real signal in the tail of the singular values, there is a certain deviation between the fitted singular values and original singular values of noise. However, under the low SNR, the deviation will be diluted due to the relatively large noise intensity. In addition, in order to reduce the influence of the deviation, this paper uses the optimal neighborhood selection strategy in Section 3.3 to carry out fault tolerance processing. 

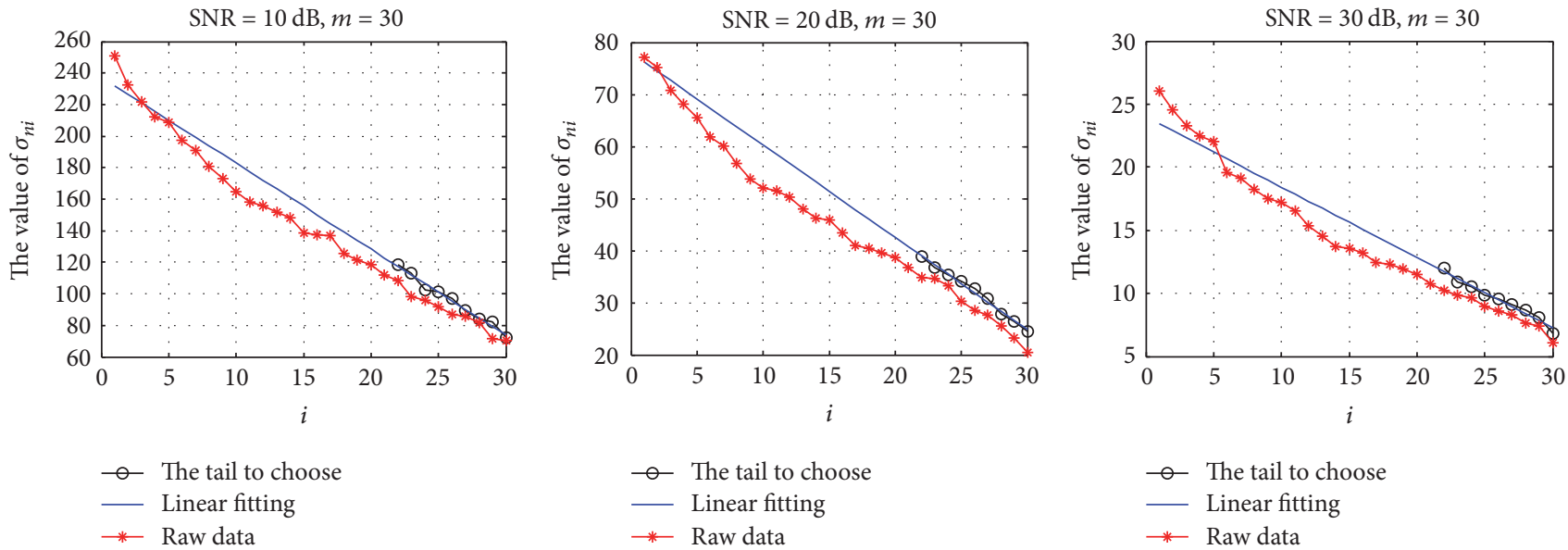

FIGURE 6: The linear fitting of the noise singular values.
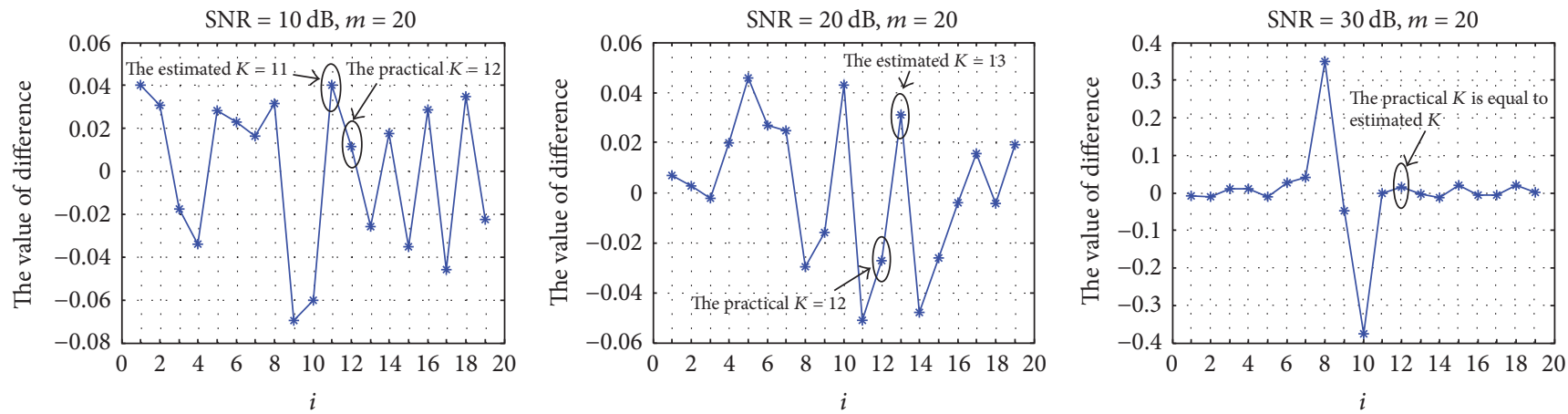

FIGURE 7: The sparsity order estimation under different SNR.

Suppose Nosie $\in \mathbb{R}^{m \times r}$ is a uniformly distributed WGN, then the intensity $I_{\text {Nosie }}$ of the noise can be calculated according to the formula

$$
\begin{aligned}
\text { Nosie } & =U S_{n} V^{T} \\
I_{\text {Noise }} & =\sqrt{\sum_{i=1}^{m} s_{n}{ }^{2}(i)},
\end{aligned}
$$

where $S_{n}=\left\{s_{n}(1), s_{n}(2), \ldots, s_{n}(i), \ldots s_{n}(m)\right\} ; s_{n}(i)$ denotes the $i$ th noise singular value.

4.2. Estimation of Sparsity Order. According to the previous analysis, we can get the estimated noise singular values $\widehat{\Sigma}_{n}=$ $\operatorname{diag}\left(\widehat{\sigma}_{n 1}, \widehat{\sigma}_{n 2}, \ldots, \widehat{\sigma}_{n m}\right)$. The contribution of noise singular values on the singular values of $Y$ is calculated by

$$
\widehat{R}_{n}=\frac{\widehat{\Sigma}_{n} \cdot}{\Sigma}=\bigcup_{i=1}^{m}\left(\frac{\widehat{\sigma}_{n i}}{\sigma_{i}}\right) .
$$

If the noise intensity is strong, $G_{R_{n}}$ is first obtained by performing gradient operation on $\widehat{R}_{n}$, and then $D_{R_{n}}$ is obtained by performing difference operation on $G_{R_{n}}$. The results of the operation are in an ascending order. The position of the minimum value in $D_{R_{n}}$ plus 1 is the estimated sparsity order $\widehat{K}$. If the sampling channel number is close to the theoretical lower limit, $\widehat{K}$ need to add one adjustable parameter $e$, and empirical value $e$ is 1 . The calculation methods of $G_{R_{n}}$ and $D_{R_{n}}$ are shown in (11). Figure 7 is a sketch of estimation of sparsity order under different SNR.

$$
\begin{aligned}
& G_{R_{n}}=\operatorname{abs}\left(\operatorname{gradient}\left(\widehat{R}_{n}, 1\right)\right), \\
& D_{R_{n}}=\operatorname{diff}\left(G_{R_{n}}\right) .
\end{aligned}
$$

When noise is low, the signal energy is dominant. The sparsity order can be estimated directly by using the singular values of $Y$. First, all singular values are shifted to the left one time, and the last empty position is filled by $\sigma_{m}$, represented as $\Sigma_{a}=\operatorname{diag}\left(\sigma_{2}, \sigma_{3}, \ldots, \sigma_{m}, \sigma_{m}\right)$. Then, $R$ is calculated by (12) and is listed in a descending order. The index of maximum value in $R$ is the estimated sparsity order $\widehat{K}$. Although $\widehat{K}$ has some deviations, the deviation has little impact on the success rate of reconstruction due to the joint sparse reconstruction and fault tolerance mechanism.

$$
R=\frac{\Sigma_{a}}{\Sigma}=\bigcup_{i=1}^{m}\left(\frac{\sigma_{a i}}{\sigma_{i}}\right)
$$




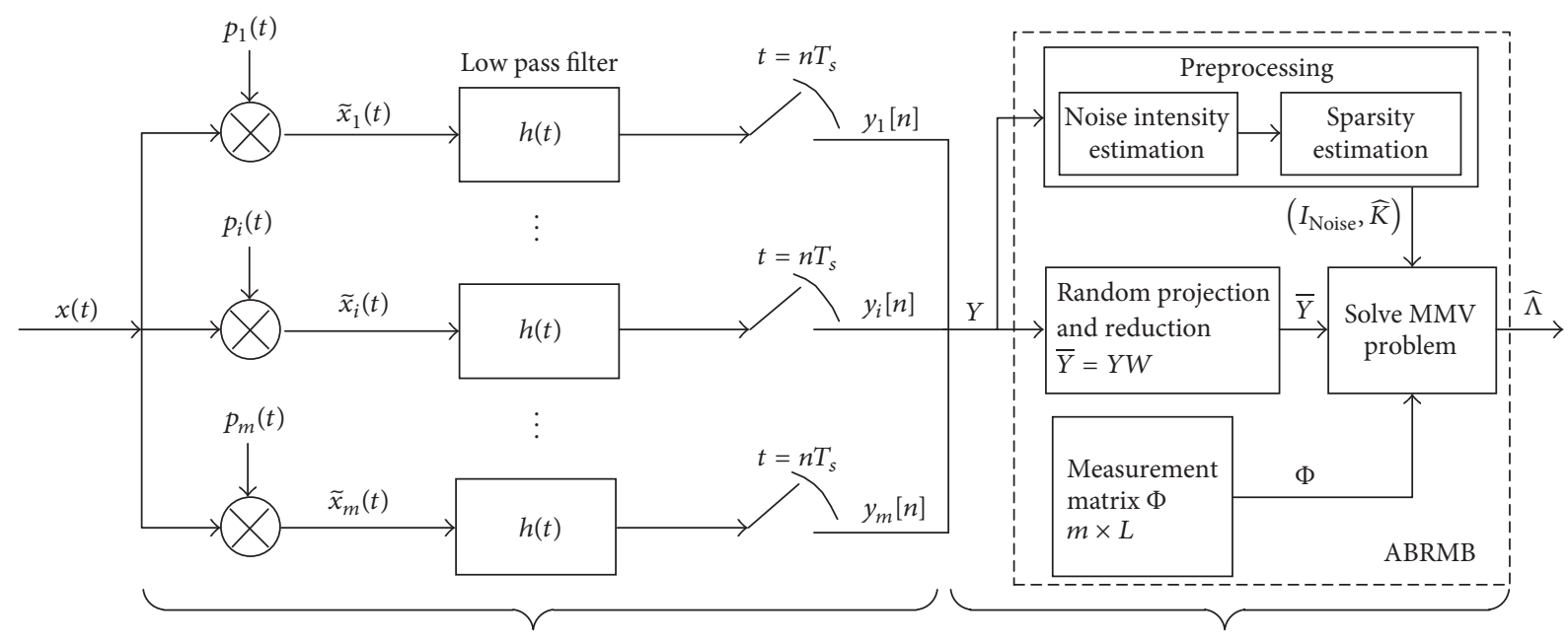

(a) MWC sub-Nyquist sampling

(b) Support reconstruction

FIGURE 8: The support reconstruction scheme based on ABRMB.

4.3. Optimal Neighborhood Selection Strategy. From the signal model showed in Figure 1(a), we know that the energy of subband can only locate on two adjacent narrow channels. If support $\Lambda_{i 1}$ is obtained, support $\Lambda_{i 2}$ can only be its neighborhood channel; that is, $\Lambda_{i 2} \in \operatorname{nei}\left(\Lambda_{i 1}\right)$. However, there are usually two neighborhood channels of $\Lambda_{i 1}$. Obviously, the channel with the largest norm 2 value is chosen to be the other support channel, as given in

$$
\Lambda_{i 2}=\operatorname{pos}\left(\max \left(\| Z^{\mathrm{nei}\left(\Lambda_{i 1}\right) \rightarrow \|_{2}}\right)\right),
$$

where the function of $\operatorname{pos}()$ is to obtain the optimal neighborhood support of $\Lambda_{i 1}$. $\left\|Z^{\text {nei }\left(\Lambda_{i 1}\right) \rightarrow}\right\|_{2}$ denotes the signal energy on two neighborhood channels of $\Lambda_{i 1}$. Obviously, this strategy is very helpful to obtain the approximate spectral support.

\section{Proposed ABRMB Optimization Scheme}

5.1. Structure of $A B R M B$ Scheme. It was pointed out in $[18,26]$ that random projection can be used for joint reconstruction to improve reconstruction performance. In particular, it can be known from [18] that the sensing performance tends to be stable if the number of preserved measured vectors is equal to $N$ after the operation of projection. The ABRMB also inherits this idea, and it is combined with the pretreatment methods mentioned in Section 3 to improve the integrated system performance. The structure of ABRMB is shown in Figure 8.

5.2. Pseudocode of $A B R M B$ Scheme. Pseudocode 1 provides the pseudocode to describe the ABRMB scheme. $W$ represents a uniformly distributed random matrix whose values are continuously selected from $[-1,1]$.

As can be seen in Pseudocode 1, the ABRMB allows a certain degree approximation deviation for spectral support with the aid of estimated noise intensity and sparsity order. In addition, if the loop iteration is ended, the optimal spectral support is still not found; the ABRMB can select the spectral support, with minimum potential, as the optimal support set from all of the stored spectral support. If there are more than one minimum potential, then choose the one with smallest residual as the best spectral support. Because of the guarantee of fault tolerance mechanism, the experiment shows that this method is very effective.

The solver of ABRMB scheme is described in Pseudocode 2.

\subsection{The Convergence of $A B R M B$ Scheme}

Theorem 1. Assume that $x(t)$ is multiband signal described as in Figure 1(a); we use the MWC structure for sample signal as shown in Figure 8. If the following conditions are established, for any $f \in\left[-f_{s} / 2, f_{s} / 2\right], z(f)$ is the only $N$-sparse solution of (4).

1. $f_{s} \geq f_{p} \geq B$, and $f_{s} / f_{p}<\left(M_{\min }+1\right) / 2$.

2. $m \geq 2 N$.

3. The number $M$ of \pm 1 symbols in a periodic sequence $p_{i}(t)$ must satisfy $M \geq M_{\min }=\left\lceil f_{\text {nyq }} / f_{p}\right\rceil$. If $f_{s}=f_{p}$, then $M_{\min }=L$.

4. Any $2 N$ column of $\Phi$ is linearly independent.

The proof of Theorem 1 is shown in [10].

Next, we prove that the spectral support of the original MMV problem $\Lambda=\operatorname{supp}(Z)$ can be obtained by solving the new MMV problem $\bar{Y}=\Phi Z$, where $\bar{Y}$ is the result after projection.

Lemma 2. Suppose $a \in R^{L}$ is a known nonzero vector, and $u \in R^{L}$ is a random set of vectors obtained from a continuous probability distribution. Then, the probability that the event " $a$ on $u$ is not 0 " is 1 . 


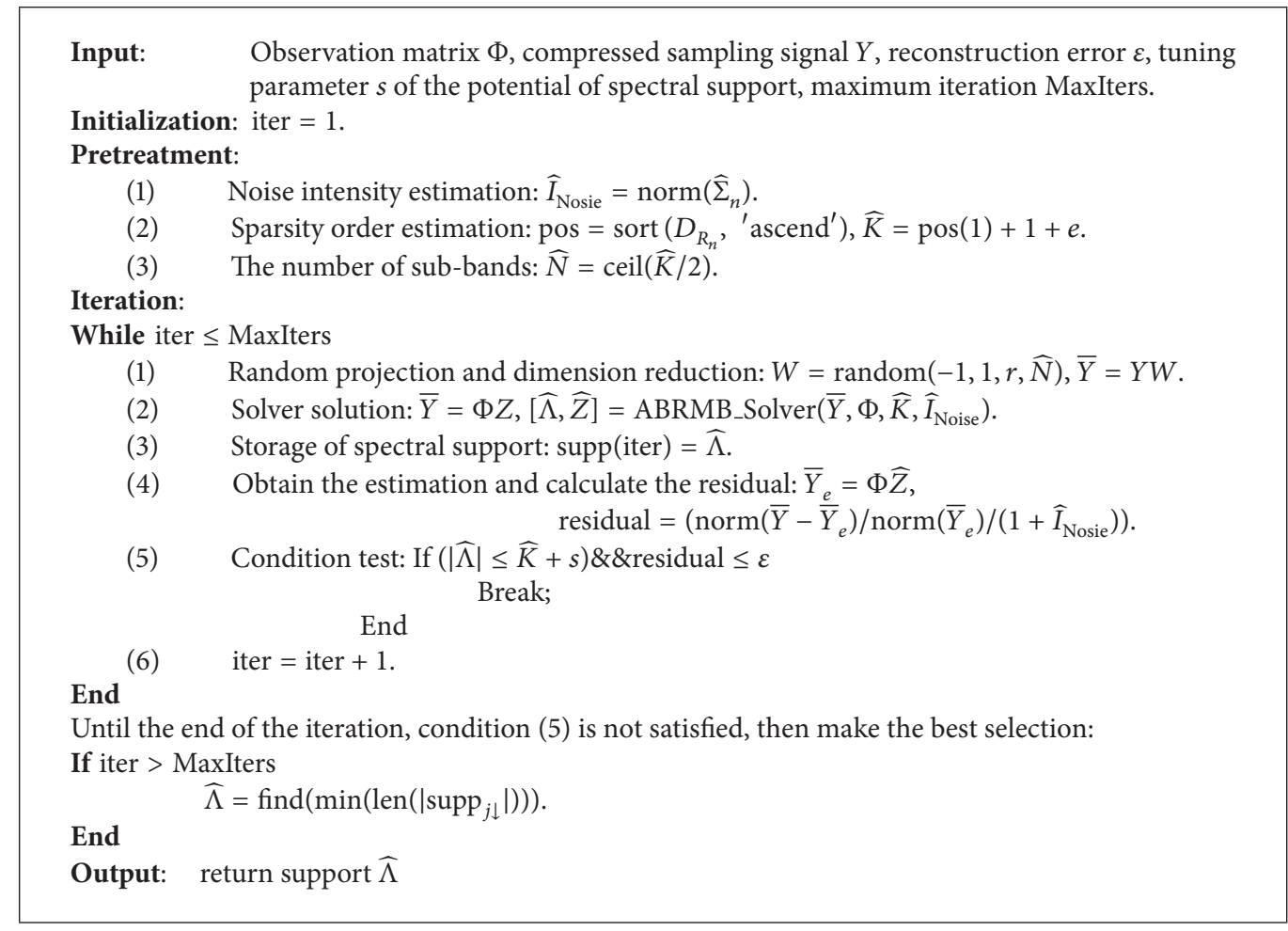

Pseudocode 1: Pseudocode of ABRMB scheme.

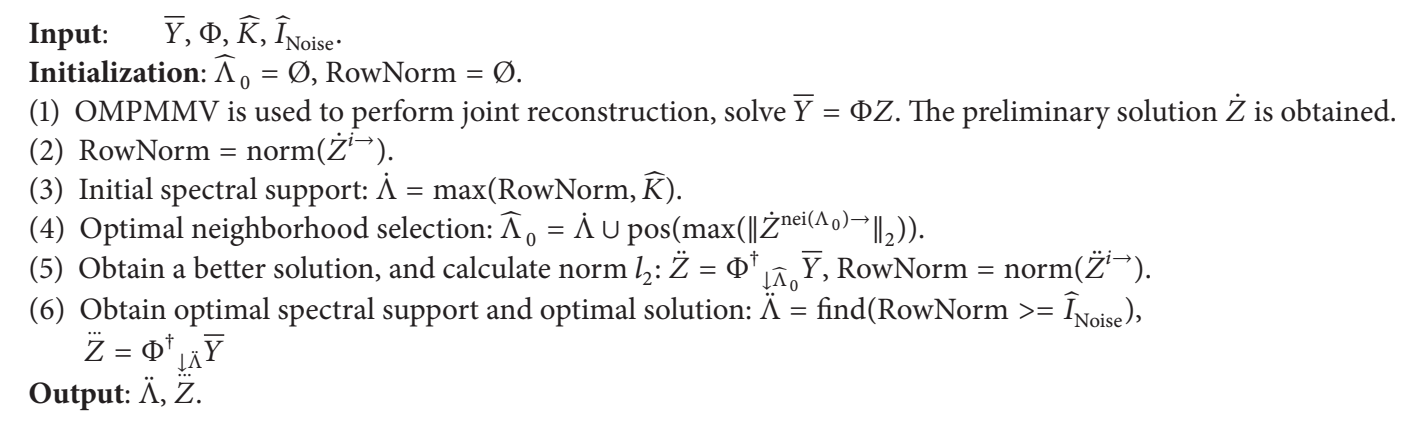

Pseudocode 2: Pseudocode of ABRMB_Solver.

Theorem 3. We know $\sigma(\Phi) \geq 2 K$, where $\sigma(\Phi)$ is the Kruskal rank of $\Phi, \widehat{Z}$ is the only $K$-sparse solution of $Y=\Phi Z$, and $W=\left\{w_{1}, w_{2}, \ldots, w_{J}\right\}(1 \leq J \leq r)$ is a random unit vector set obtained from a continuous probability distribution, whose length is $r$. Suppose $\bar{Y}=Y W, \bar{Z}=\widehat{Z} W$, considering $a$ new $M M V$ problem $\bar{Y}=\Phi Z$ after dimension reduction, the probability of $\operatorname{supp}(\widehat{Z})=\operatorname{supp}(\bar{Z})$ is 1. Meanwhile, $\bar{Z}$ is the only K-sparse solution. [18].

The proof of Lemma 2 and Theorem 3 can be found in

Thus, Theorem 1, Lemma 2, and Theorem 3 can guarantee the convergence of the proposed scheme.

\section{Numerical Results and Performance Analysis}

\subsection{Performance Evaluation Indices}

6.1.1. The Required Minimum Number of Sampling Channels and Minimum Sampling Rate. When the required conditions are met, it is pointed out in [10] that the spectral support of the signal can be reconstructed as long as the number of sampling channels is satisfied by $m \geq 2 N$ in theory. However, the existing algorithms are difficultly reach the theoretical lower limit and can only approximate the theoretical value by continuously improving the algorithms. 
In addition, MWC can achieve sub-Nyquist sampling. Without affecting the success rate of reconstruction, the subsequent processing of the system is more favorable with higher degree of compression. The total sampling rate of MWC is $f_{\Sigma}=m f_{s}$. The theoretical minimum of sampling rate for multiband signal, that is, the Landau rate [15], is defined as

$$
M\left(P_{N}\right)=2 \sum_{i=1}^{N / 2}\left(b_{i}-a_{i}\right)
$$

where $M\left(P_{N}\right)$ is Landau rate; that is, it is the sum of all subband frequency widths, $P_{N}$ denotes the union of $N$ effective frequency components in multiband signal, and $\left(b_{i}-\right.$ $a_{i}$ ) is frequency width of $i$ th subband.

Since the number of the channels $m$ and total sampling rate $f_{\Sigma}$ are directly relevant, obviously, the cost of the system and the corresponding sampling rate are lower for smaller $m$.

6.1.2. The Maximum Number of Subbands That Can Be Reconstructed in Signal. From [16], the upper bound of the reconstruction capability of the MMV problem is

$$
I(Z) \leq \frac{(\sigma(\Phi)+\operatorname{Rank}(Y)-1)}{2} .
$$

In (15), $I(Z)$ is the joint sparsity order of $Z, \sigma(\Phi)$ is the Kruskal rank of $\Phi$, and $\operatorname{Rank}(Y)$ is the rank of $Y$. As can be learned from (15), the smaller the rank $Y$, the lower the sparsity order of the signal that can be reconstructed, which is also the reason that the upper bound of the sparsity order in [15] is particularly small. The number of subbands is $N=$ $I(Z) / 2$. Therefore, the performance of the system is better when the number of subbands that can be reconstructed is larger.

6.1.3. The Approximate Error of the Support Potential. For the original signal, the potential of spectral support is $|\Lambda|$, that is, the length of spectral support. Obviously, $|\Lambda|=\|\Lambda\|_{0}$. If $|\widehat{\Lambda}|=|\Lambda|$, then the approximate error is 0 . If $|\widehat{\Lambda}|>|\Lambda|$, then the approximate error existed. In this paper, the approximate error $E_{\widehat{\Lambda}}$ and the upper bound of the error $E_{\text {upper }}$ are defined as

$$
\begin{aligned}
E_{\widehat{\Lambda}} & =\frac{(|\widehat{\Lambda}|-|\Lambda|)}{L} \text { s.t. }|\widehat{\Lambda}|>|\Lambda| \\
E_{\text {upper }} & =\frac{|\Lambda|_{\max }}{L} .
\end{aligned}
$$

In (16), $|\widehat{\Lambda}|$ is the potential of estimated support, $(|\widehat{\Lambda}|-$ $|\Lambda|)$ represents the difference between the estimated support potential and the actual support potential, and "s.t." is the abbreviation of "subject to". In (17), $|\Lambda|_{\max }$ is the potential of maximum spectral support for multiband sparse signals. $L$ is the number of narrow bands, and $N$ is the number of support bands of the original signal. Apparently, smaller approximate error is better. Since $L \gg|\Lambda|$, in the application of wideband spectrum sensing in cognitive radio, as long as $E_{\widehat{\Lambda}} \leq E_{\text {upper }}$, the impact on the secondary users can be negligible.
6.1.4. The Successful Probability of the Reconstruction. In the analysis of recovered spectral support, we refer to the successful recovery criteria in [10]; that is, when the estimated support $\widehat{\Lambda}$ and the actual support $\Lambda$ satisfy (18), where $\widehat{\Lambda} \supseteq$ $\Lambda$, and $\Phi_{\downarrow \widehat{\Lambda}}$ is with full column rank, it is considered as a successful reconstruction. In the experiment of this paper, reconstruction is performed 500 times in the same simulation environment. If the success rate of reconstruction is more than $90 \%$, it is considered a high probability reconstruction.

$$
\begin{aligned}
& \text { success } \\
& \text { s.t. }\left(E_{\widehat{\Lambda}} \leq E_{\text {upper }} \& \& \widehat{\Lambda} \supseteq \Lambda \& \& \operatorname{Rank}\left(\Phi_{\downarrow \widehat{\Lambda}}\right)=\|\widehat{\Lambda}\|_{0}\right) .
\end{aligned}
$$

6.2. Experimental Analysis. In order to verify the effectiveness of the proposed scheme, simulation experiments are carried out to analyze the system performance metrics, including minimum number of sampling channels, the success rate of reconstruction, and the maximum number of subbands that can be reconstructed. Then, these metrics are used to compare with ReMBo and PRMB algorithms. Meanwhile, the approximate error of support potential is also analyzed.

The sparse multiband analog signal with noise is generated from sinc signal by

$$
\begin{aligned}
x(t)= & \sum_{i=1}^{N / 2} \sqrt{E_{i} B_{i}} \operatorname{sinc}\left(B_{i}\left(t-\tau_{i}\right)\right) \cos \left(2 \pi f_{i}\left(t-\tau_{i}\right)\right) \\
& +n(t) .
\end{aligned}
$$

In (19), $E_{i}, B_{i}, f_{i}$, and $\tau_{i}$ represent the energy coefficient, bandwidth, carrier frequency, and time offset of the $i$ th band, respectively. $N$ is the number of subbands in the signal. $n(t)$ is white Gaussian noise. The following procedure is repeated 500 times to calculate the probability of success.

1. Generate the mixing signal $p_{i}(t)$ randomly.

2. Generate the carrier frequency $f_{i}$ uniformly and randomly in $\left[-f_{\text {nyq }} / 2, f_{\text {nyq }} / 2\right]$.

3. Generate new sinc signal according to $f_{i}$.

4. Estimate the spectral support using ReMBo, RPMB, and $A B R M B$, respectively, and determine whether it is successfully recovered.

In the simulation, the settings of parameters of the signal are $N=6$ (3 balanced pairs); $E_{i} \in\{1,2,3\}, B_{i} \in$ $\{50,50,50\} \mathrm{MHz}, \tau_{i} \in\{0.4,0.7,0.2\} \mu \mathrm{s}$, carrier frequency $f_{i}$ randomly distributed in the interval $\left[-f_{\text {nyq }} / 2, f_{\text {nyq }} / 2\right]$, and $f_{\text {nyq }}=10 \mathrm{GHz}$. The parameters of MWC sampling are $L_{0}=$ 97, $L=2 L_{0}+1=195, f_{s}=f_{p}=f_{\text {nyq }} / L=51.28 \mathrm{MHz}$, number of channels $m$ steps using 1 as interval in the range $[15,40]$, and MaxIters $=20$. The noise is WGN, and SNR $=$ $\{10,20,30\} \mathrm{dB}$. The other parameters are $\varepsilon=0.001, s=2$.

Firstly, under the same conditions, the changes of the success rate of reconstruction with channel number using $\mathrm{ABRMB}, \mathrm{RPMB}$, and ReMBo are studied under different SNR. As can be seen in Figure 9, when SNR equals 10, 20, and 30 , respectively, the success rate of the reconstruction of 


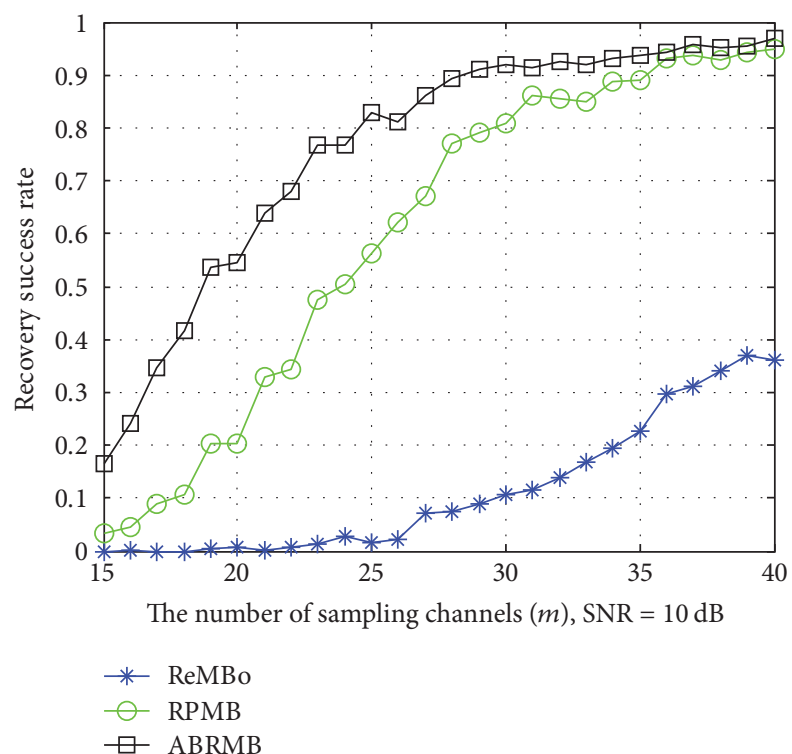

(a)

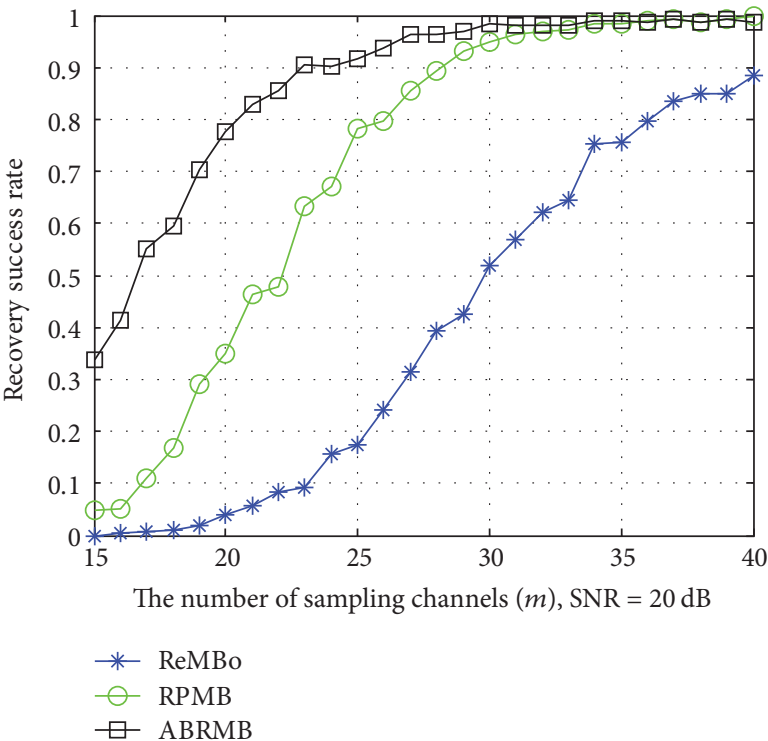

(b)

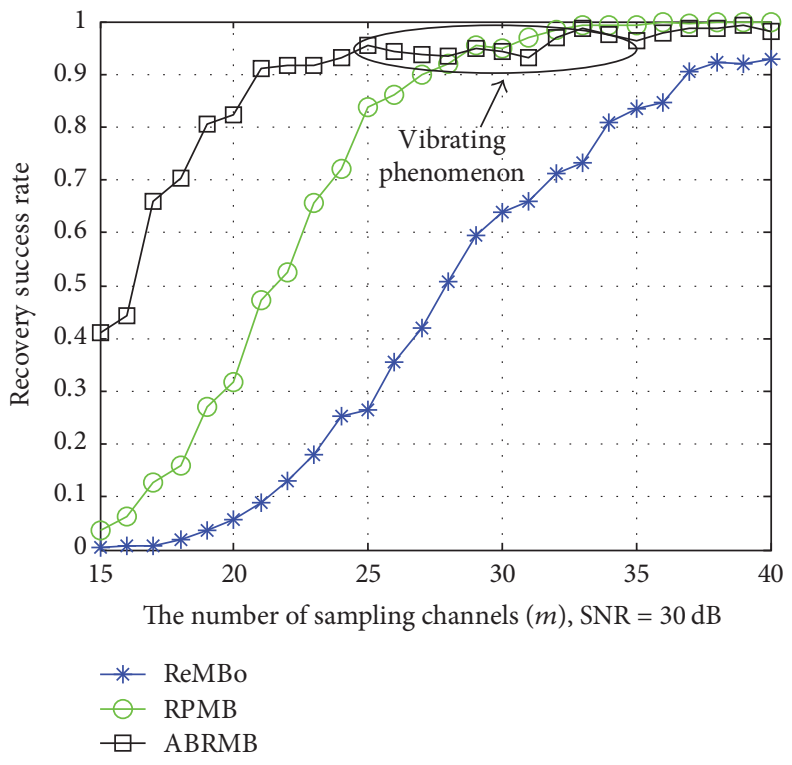

(c)

FIGURE 9: The comparison of recovery success rate of support under different SNR.

spectral support using ABRMB is better than using ReMBo or RPMB. Particularly, when $m=20$ and SNR $=\{10,20,30\} \mathrm{dB}$, the improvements of the success probability of $A B R M B$ compared with RPMB and ReMBo are shown in Table 1. Therefore, ABRMB scheme is more effective and has better adaptability in the case when SNR is low or fluctuated due to the reasonable estimation of the noise intensity and the sparsity order and the optimal neighborhood selection strategy.

Secondly, in the same circumstances, the needed minimum channel number and the minimum sampling rate of high probability reconstruction are studied. As shown in Figure 9 and Table 2, under different SNR, the number of hardware channels and the sampling rate of ABRMB for the
TABLE 1: Comparison of maximum promoting rate of the reconstruction success probability.

\begin{tabular}{lccc}
\hline Comparison & \multicolumn{3}{c}{$m=20$} \\
& $\mathrm{SNR}=10 \mathrm{~dB}$ & $\mathrm{SNR}=20 \mathrm{~dB}$ & $\mathrm{SNR}=30 \mathrm{~dB}$ \\
\hline ABRMB versus RPMB & $34.2 \% \uparrow$ & $42.8 \% \uparrow$ & $50.8 \% \uparrow$ \\
ABRMB versus ReMBo & $53.6 \% \uparrow$ & $73.6 \% \uparrow$ & $76.6 \% \uparrow$ \\
\hline
\end{tabular}

high probability reconstruction are smaller than those of the ReMBo and RPMB. Thus, our proposed scheme can use fewer hardware channels and lower sampling rate to achieve the high success rate of reconstruction. Obviously, the cost of 
TABLE 2: The comparison of the minimum channel and minimum sampling rate needed.

\begin{tabular}{lcccccc}
\hline \multirow{2}{*}{ Algorithms } & \multicolumn{2}{c}{$\mathrm{SNR}=10 \mathrm{~dB}$} & \multicolumn{2}{c}{$\mathrm{SNR}=20 \mathrm{~dB}$} & \multicolumn{2}{c}{$\mathrm{SNR}=30 \mathrm{~dB}$} \\
& $m_{\min }$ & $f_{\sum \min } / \mathrm{MHz}$ & $m_{\min }$ & $f_{\sum \min } / \mathrm{MHz}$ & $m_{\min }$ & $f_{\sum \min } / \mathrm{MHz}$ \\
\hline ABRMB & 29 & 1487.12 & 23 & 1179.44 & 21 & 1076.88 \\
ReMBo & - & - & 40 & 2051.2 & 37 & 1897.36 \\
RPMB & 36 & 1846.08 & 28 & 1435.84 & 27 & 1384.56 \\
\hline
\end{tabular}

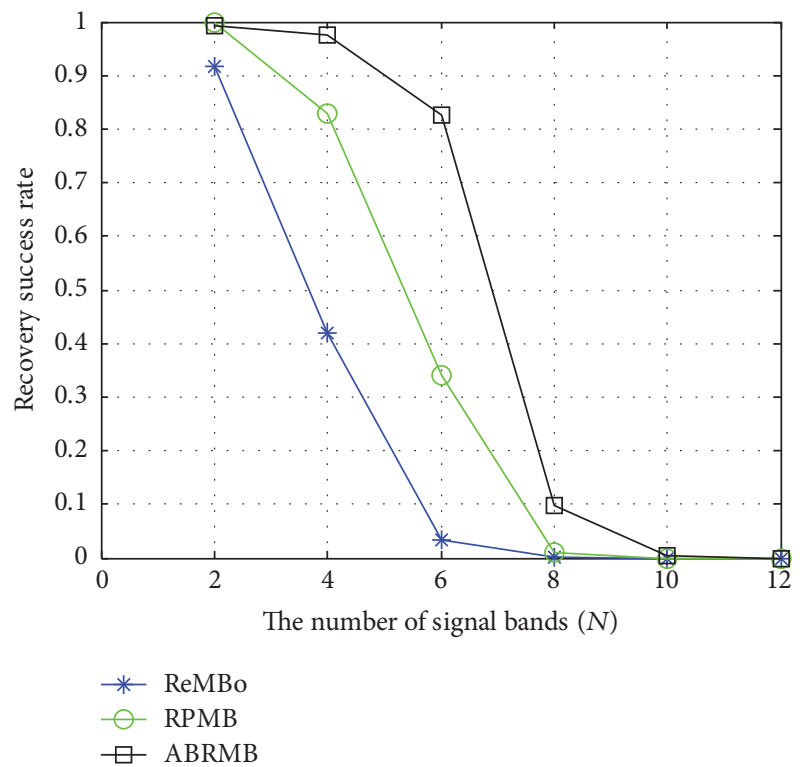

FIGURE 10: The effects of subbands number on the support recovery.

the system can be saved as the number of hardware channels needed by the scheme which can be reduced.

Next, under the same conditions, the changes of success rate of reconstruction with the number of the bands are studied using these three algorithms. The number of the subbands $N$ is chosen in the range $[2,12]$ with 2 as its advancing step. The parameters are SNR $=20 \mathrm{~dB}, m=20$, $E_{i} \in\{1,2,3,4,5,6\}$, and $\tau_{i} \in\{0.4,0.7,0.2,0.9,1.2,1.5\} \mu \mathrm{s}$. As shown in Figure 10, since the number of the bands is directly related to the sparsity order of the signal, with the increase of $N$, the signal is not sparse enough and the success rate of reconstruction for three algorithms is reduced. Nevertheless, the reconstruction performance of $A B R M B$ is obviously better than that of RPMB and ReMBo for $N=4,6$, and 8 .

At last, we compare the average approximation error on the potential of estimated spectral support. We set $N=6$, SNR $=20 \mathrm{~dB}$, and $m$ is in the range $[15,40]$ with 1 as its step. As can be seen in Figure 11, since the potential of the support reconstructed by RPMB is related to the number of channels, when $m=24$, the average approximation error of the spectral support of RPMB has already reached the upper limit. From Figure 11, the average approximation error of ABRMB is the smallest in these three algorithms, and the potential of the spectral support reconstructed by ABRMB is the closest to the actual number of frequency channels. In this way, the

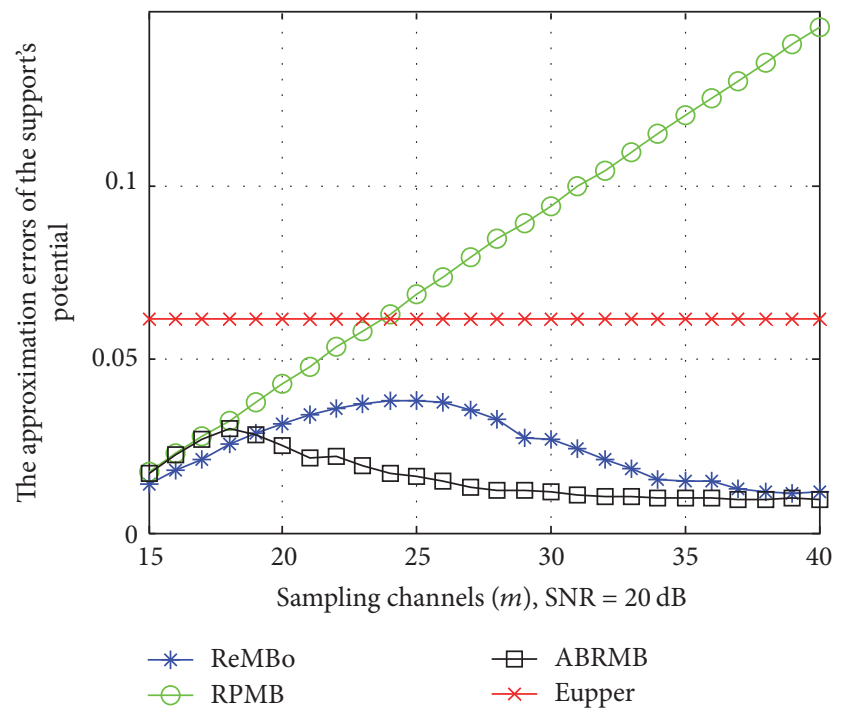

Figure 11: The comparison of the approximation error of the support potential.

ABRMB can provide more spectrum access opportunities for the secondary users in cognitive radio networks.

From the analysis of above four performance metrics, we can see that ABRMB scheme can achieve low-speed sampling by utilizing the sparse characteristics of wideband signals in frequency domain. The sampling rate can be reduced to $14.9 \%$ of the Nyquist sampling rate when SNR $=10 \mathrm{~dB}$. Meanwhile, for noise uncertainty, the detection threshold can be adaptively changed as the noise power changes in ABRMB scheme. Based on the neighborhood selection strategy and sparsity estimation, ABRMB can obtain higher success reconstruction probability. Then, the projection reduction operation can reduce the computational complexity, and it does not affect the convergence of the scheme. Finally, ABRMB scheme can find better trade-off between noise interference and sensing accuracy.

\section{Conclusions}

By using SVD theory, this paper proposes a self-adaptive and blind wideband spectrum sensing MWC scheme which leads to a flexible and high performance solver. The SVD is performed before signal reconstruction in the preprocessing block. The noise intensity and sparsity order of the signal are estimated in this block, and then the subbands number of the signal can be obtained. ABRMB scheme can use the estimated noise intensity and sparsity order to process the multiband 
signals adaptively, and it improves the reconstruction performance by using optimal neighborhood selection. In practical sensing environment, especially for low or fluctuated SNR, the reconstruction of the spectral support can be performed more accurately by using the ABRMB. The simulation results demonstrate that, compared with ReMBo algorithm and RPMB algorithm, the reconstruction performance has been improved by $34.2-76.6 \%$; the minimum number of hardware channels and minimum sampling rate needed by high probability reconstruction have been decreased by $17.9-22.2 \%$. When we perform reconstruction of spectral support on the signal with different number of subbands, the performance of ABRMB is better than the other two algorithms. In the study of the approximation error of the support potential by using the three algorithms, the approximation error of ABRMB is the smallest.

\section{Notations}

$\Lambda: \quad$ The actual spectral support of signal

$\dot{\Lambda}: \quad$ The obtained initial support in

ABRMB_Solver

$\ddot{\Lambda}$ : $\quad$ The optimal solution in

ABRMB_Solver

$\widehat{\Lambda}: \quad$ The obtained spectral support with ABRMB

$|\Lambda|: \quad$ The potential of spectral support

$N$ : $\quad$ The number of subbands in multiband signals

$B_{i}$ : $\quad$ Bandwidth of $i$ th subband

$f_{\text {nyq }}$ : Nyquist rate of $x(t)$

$p_{i}(t): \quad$ Periodic mixing signal

$Y: \quad$ The sub-Nyquist sampling signal with MWC

$\bar{Y}$ : $\quad$ The sampling matrix after projection

$K$ : $\quad$ Sparsity order of signal

$\widehat{K}: \quad$ The estimated sparsity order

$\left\|\widetilde{Z}^{i \rightarrow}\right\|_{2}$ : The norm of each row vector for $\widetilde{Z}$

$\Phi: \quad m \times L$ measurement matrix or observation matrix

$\Phi_{\downarrow}^{\dagger}: \quad$ Extracting column vectors from $\Phi$ according to $\ddot{\Lambda}$ and then conducting pseudoinverse operation

$\varepsilon$ : $\quad$ The decision threshold

$I_{\text {Nosie }}$ : The noise intensity

$E_{i}$ : $\quad$ The energy coefficient of $i$ th subband

$f_{i}: \quad$ Carrier frequency

$\tau_{i}: \quad$ The time offset of $i$ th subband

ceil: The function of round toward positive infinity

L: $\quad$ Spectrum slice number

$f_{p}: \quad$ Spectral slice width, $f_{p}=f_{\text {nyq }} / L$

$f_{s}$ : Sampling rate at each channel, $f_{s}=q f_{p}$, with odd $q$.

\section{Conflicts of Interest}

The authors declare that they have no conflicts of interest.

\section{Acknowledgments}

This work was supported by the National Natural Science Foundation of China (61561017), the Hainan Province Natural Science Foundation of China (617033, 20166232), the Open Sub-Project of State Key Laboratory of Marine Resource Utilization in South China Sea (2016013B), Oriented Project of State Key Laboratory of Marine Resource Utilization in South China Sea (DX2017012), and the Major Science and Technology Project of Hainan Province (no. ZDKJ2016015).

\section{References}

[1] H. Sun, A. Nallanathan, C. Wang, and Y. Chen, "Wideband spectrum sensing for cognitive radio networks: a survey," IEEE Wireless Communications Magazine, vol. 20, no. 2, pp. 74-81, 2013.

[2] A. Furtado, L. Irio, R. Oliveira, L. Bernardo, and R. Dinis, "Spectrum sensing performance in cognitive radio networks with multiple primary users," IEEE Transactions on Vehicular Technology, vol. 65, no. 3, pp. 1564-1574, 2016.

[3] A. A. Alkheir and M. Ibnkahla, "A selective decision-fusion rule for cooperative spectrum sensing using energy detection," Wireless Communications and Mobile Computing, vol. 16, no. 12, pp. 1603-1611, 2016.

[4] S. K. Sharma, E. Lagunas, S. Chatzinotas, and B. Ottersten, "Application of compressive sensing in cognitive radio communications: a survey," IEEE Communications Surveys and Tutorials, vol. 18, no. 3, pp. 1838-1860, 2016.

[5] M. Yang, Y. Li, X. Liu, and W. Tang, "Cyclostationary feature detection based spectrum sensing algorithm under complicated electromagnetic environment in cognitive radio networks," China Communications, vol. 12, no. 9, Article ID 7275257, pp. 35-44, 2015.

[6] K. Cichon, A. Kliks, and H. Bogucka, "Energy-efficient cooperative spectrum sensing: a survey," IEEE Communications Surveys and Tutorials, vol. 18, no. 3, pp. 1861-1886, 2016.

[7] M. J. Saber and S. M. S. Sadough, "Multiband cooperative spectrum sensing for cognitive radio in the presence of malicious users," IEEE Communications Letters, vol. 20, no. 2, pp. 404-407, 2016.

[8] Z. Tian and G. B. Giannakis, "Compressed sensing for wideband cognitive radios," in Proceedings of the IEEE International Conference on Acoustics, Speech and Signal Processing (ICASSP '07), pp. 1357-1360, April 2007.

[9] E. J. Candes and M. B. Wakin, "An introduction to compressive sampling: A sensing/sampling paradigm that goes against the common knowledge in data acquisition," IEEE Signal Processing Magazine, vol. 25, no. 2, pp. 21-30, 2008.

[10] M. Mishali and Y. C. Eldar, "From theory to practice: subnyquist sampling of sparse wideband analog signals," IEEE Journal of Selected Topics in Signal Processing, vol. 4, no. 2, pp. 375-391, 2010.

[11] M. Mishali, Y. C. Eldar, O. Dounaevsky, and E. Shoshan, "Xampling: analog to digital at sub-Nyquist rates," IET Circuits, Devices and Systems, vol. 5, no. 1, pp. 8-20, 2011.

[12] Y. C. Eldar, R. Levi, and A. Cohen, "Clutter removal in subNyquist radar," IEEE Signal Processing Letters, vol. 22, no. 2, pp. 177-181, 2015. 
[13] M. A. Lexa, M. E. Davies, and J. S. Thompson, "Reconciling compressive sampling systems for spectrally sparse continuoustime signals," IEEE Transactions on Signal Processing, vol. 60, no. 1, pp. 155-171, 2012.

[14] D. Cohen, A. Akiva, B. Avraham, S. Patterson, and Y. C. Eldar, "Distributed cooperative spectrum sensing from subNyquist samples for Cognitive Radios," in Proceedings of the 16th IEEE International Workshop on Signal Processing Advances in Wireless Communications, SPAWC '15, pp. 336-340, IEEE, Stockholm, Sweden, July 2015.

[15] M. Mishali and Y. C. Eldar, "Reduce and boost: recovering arbitrary sets of jointly sparse vectors," IEEE Transactions on Signal Processing, vol. 56, no. 10, part 1, pp. 4692-4702, 2008.

[16] J. Chen and X. Huo, "Theoretical results on sparse representations of multiple-measurement vectors," IEEE Transactions on Signal Processing, vol. 54, no. 12, pp. 4634-4643, 2006.

[17] H. J. Landau, "Necessary density conditions for sampling and interpolation of certain entire functions," Acta Mathematica, vol. 25, no. 2, pp. 37-52, 1967.

[18] J. X. Gai, P. Fu, J. Y. Sun, H. J. Lin, and L. H. Wu, "A recovery algorithm of MWC sub-Nyquist sampling based on random projection method," Acta Electronica Sinica, vol. 42, no. 9, pp. 1686-1692, 2014.

[19] Z. Drmac, "Accurate computation of the product-induced singular value decomposition with applications," SIAM Journal on Numerical Analysis, vol. 35, no. 5, pp. 1969-1994, 1998.

[20] X. Yang, X. Tao, Q. Cui, and Y. J. Guo, "nterference-constrained adaptive simultaneous spectrum sensing and data transmission scheme for unslotted cognitive radio network," EURASIP Journal on Wireless Communications and Networking, vol. 2012, article 102, 2012.

[21] N. Hasan, H. Kim, W. Ejaz, and S. Lee, "Knapsack-based energyefficient node selection scheme for cooperative spectrum sensing in cognitive radio sensor networks," IET Communications, vol. 6, no. 17, pp. 2998-3005, 2012.

[22] W. Ejaz, G. A. Shah, N. U. Hasan, and H. S. Kim, "Energy and throughput efficient cooperative spectrum sensing in cognitive radio sensor networks," Transactions on Emerging Telecommunications Technologies, vol. 26, no. 7, pp. 1019-1030, 2015.

[23] Z. H. Hu, Y. Bai, Y. C. Zhao, and Y. R. Zhang, "Support recovery for multiband spectrum sensing based on modulated wideband converter with SwSOMP algorithm," in Proceedings of the 1st EAI International Conference on $5 G$ for Future Wireless Networks, 2017.

[24] W. Sun, Z. Huang, F. Wang, and X. Wang, "Compressive wideband spectrum sensing based on single channel," IEEE Electronics Letters, vol. 51, no. 9, pp. 693-695, 2015.

[25] P. Yang, Z.-T. Huang, Z. Liu, and W.-L. Jiang, "Single-channel spectrum sensing technique based on sub-Nyquist sampling," in Proceedings of the 2012 11th International Conference on Signal Processing, ICSP '12, pp. 224-227, October 2012.

[26] S. F. Cotter, B. D. Rao, K. Engan, and K. Kreutz-Delgado, "Sparse solutions to linear inverse problems with multiple measurement vectors," IEEE Transactions on Signal Processing, vol. 53, no. 7, pp. 2477-2488, 2005. 


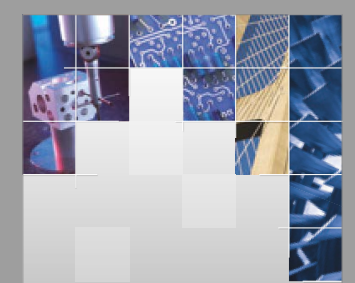

\section{Enfincering}
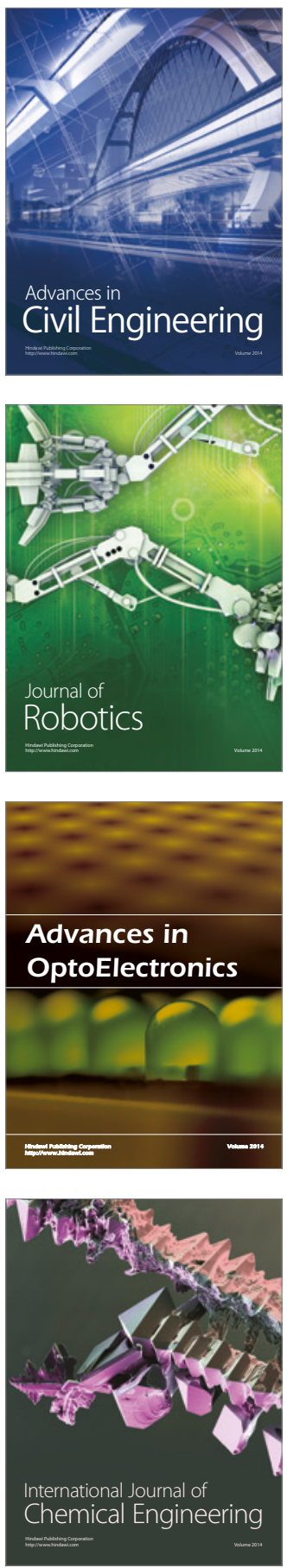

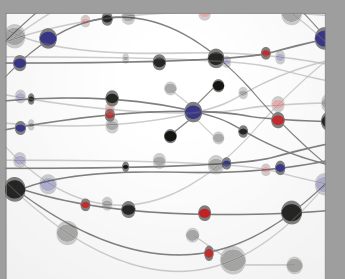

The Scientific World Journal

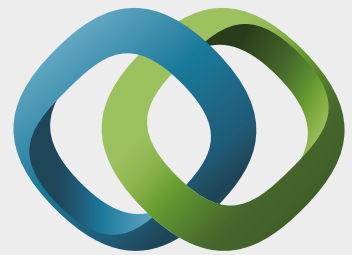

\section{Hindawi}

Submit your manuscripts at

https://www.hindawi.com
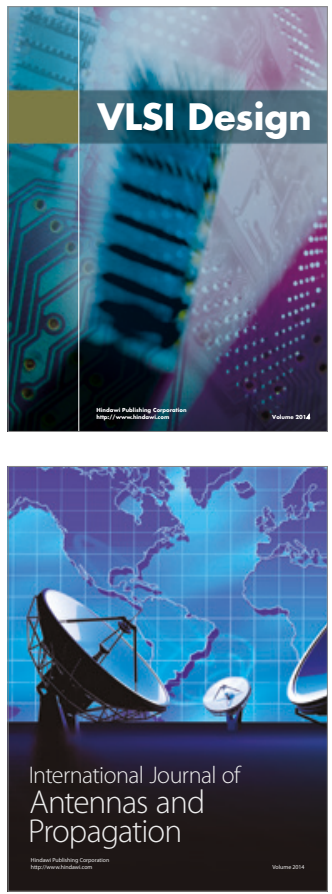

\section{Rotating}

Machinery
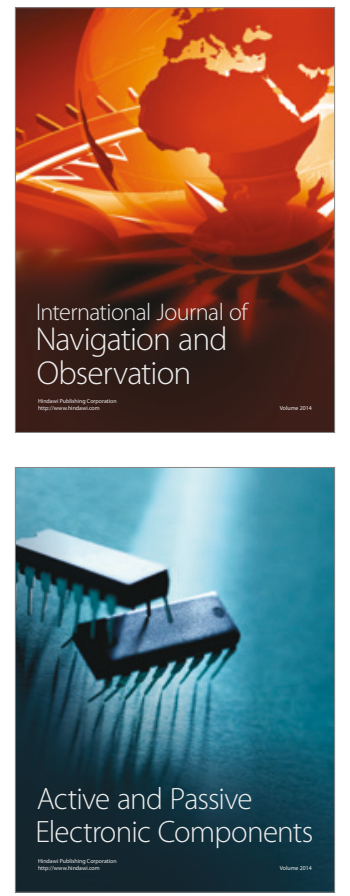
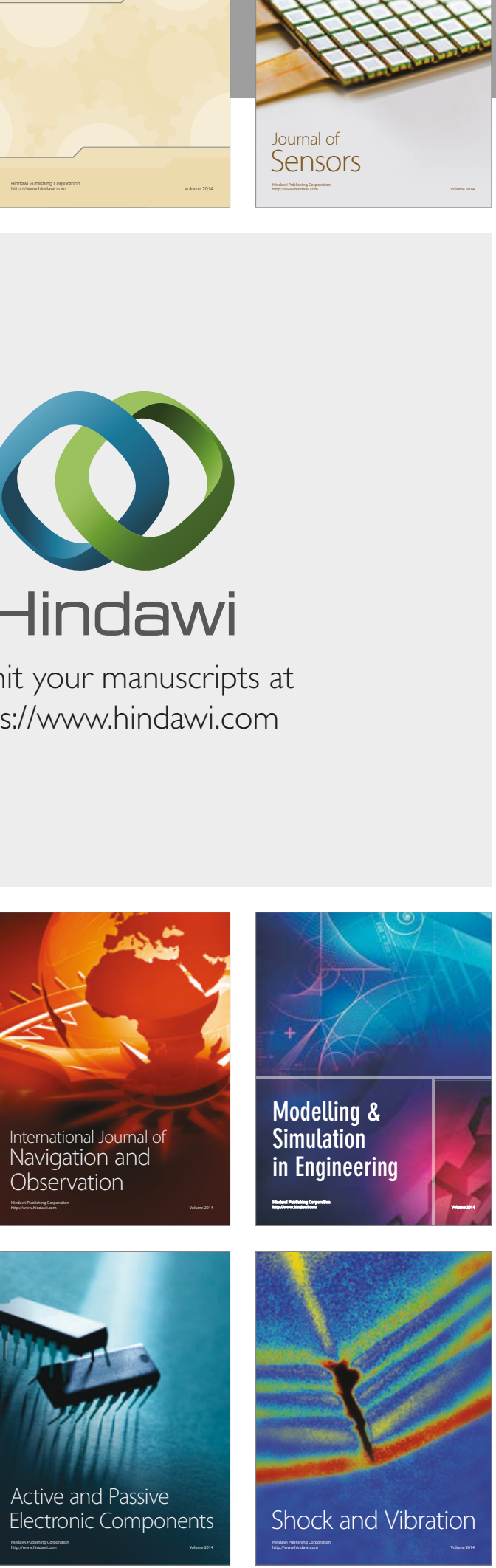
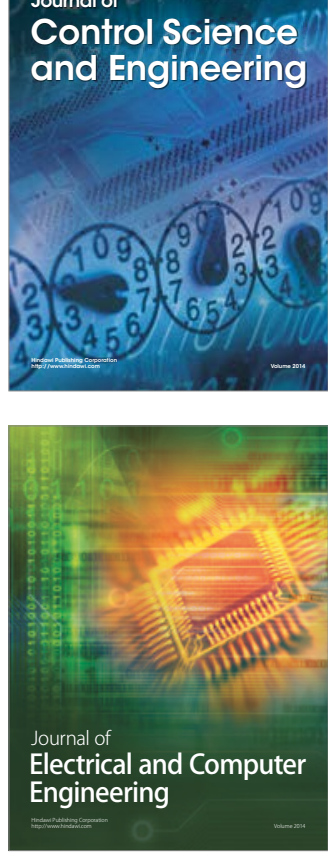

Distributed

Journal of

Control Science

and Engineering
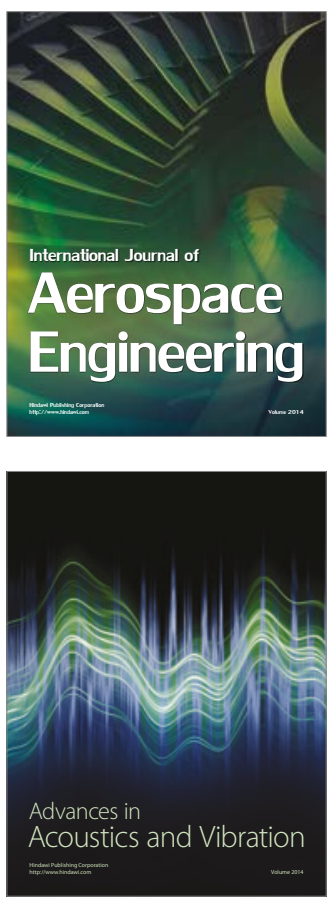

Sensor Networks 